\title{
ANÁLISIS COSTO-BENEFICIO EN LA NORMATIVA AMBIENTAL CHILENA BAJO LA LEY 19.300*
}

\author{
Ricardo Katz, Guillermo González \\ y María Gabriela Cienfuegos
}

\begin{abstract}
La Ley 19.300 introdujo formalmente el análisis económico en los procesos de elaboración de ciertas regulaciones ambientales en Chile, además de generar procedimientos reglamentados, intersectoriales y participativos. Eso significó un importante avance. En este trabajo se procura visualizar dónde podría utilizarse potencialmente el análisis costo-beneficio (ACB) a lo largo del proceso de elaboración de las distintas normativas. Se sostiene que el ACB puede servir para definir qué normas quedarán seleccionadas en el Programa Priorizado de Normas. En el caso de las normas de calidad ambiental, al no existir estándares de riesgo definidos políticamente, el ACB podría convertirse en un poderoso instrumento técnico para definir los niveles de concentración óptimos. En cuanto a las normas
\end{abstract}

Ricardo Katz. Ingeniero Civil, Universidad de Chile. Máster en Ciencias en Administración Ambiental, Universidad de Texas. Investigador asociado y coordinador de la Comisión de Medio Ambiente del CEP.

Guillermo GonzÁLEz. Ingeniero Civil mención en Ingeniería Ambiental, Universidad Católica de Chile. Especialista ambiental de la consultora GAC.

María Gabriela Cienfuegos. Ingeniera Ambiental, Universidad de Valparaíso. Especialista ambiental de la consultora GAC.

* Una versión preliminar fue presentada en el seminario "El desafío del análisis costo-beneficio ambiental”, organizado por el CEP el 17 de junio de 2009. 
de emisión, cuando éstas constituyen instrumentos para alcanzar niveles de concentración establecidos en una cierta norma de calidad ambiental, bastaría con realizar un análisis de costoefectividad (ACE) entre las distintas alternativas existentes. El caso de los planes de prevención y descontaminación es análogo a éste, pues el ACE serviría para determinar el conjunto de medidas que permitirán alcanzar la meta ambiental al menor costo.

Respecto al Sistema de Evaluación de Impacto Ambiental, como éste no permite que los proyectos se hagan cargo de sus impactos ambientales mediante transacciones económicas, se señala que eso podría explicar en parte por qué las comunidades locales a veces se oponen a la aprobación de los proyectos. Por otro lado, al importar directamente normas extranjeras de referencia (cuando no existen normas nacionales) para la evaluación ambiental de proyectos, se produce una asimetría en términos de la evaluación económica subyacente.

Por último, se realiza un estudio de los AGIES (Análisis General del Impacto Económico y Social) realizados a la fecha, donde se constata una importante divergencia en las metodologías utilizadas en esos análisis.

Palabras clave: análisis costo-beneficio; análisis costo-efectividad; normativa ambiental; sistema de evaluación de impacto ambiental.

\section{Introducción}

ت análisis de costos y beneficios en la generación de normativas ambientales ${ }^{1}$ se inició formalmente en Chile con la promulgación de la Ley 19.300 de Bases Generales del Medio Ambiente en 1994. Antes de eso, la normativa ambiental no era evaluada desde una perspectiva económica, por lo menos de modo formal. Es más, en Chile la mezcla de análisis económico con conservación ambiental era considerada un pecado por los grupos ambientalistas y también, aunque en menor medida, por los profesionales que trabajaban en temas ambientales, y los especialistas que utilizaban estos instrumentos eran calificados de "economicistas".

${ }^{1}$ Normativa ambiental entendida en el contexto de las definiciones de la Ley 19.300 y que es el sujeto de evaluación costo-beneficio (básicamente norma de control de la contaminación atmosférica, hídrica, acústica y lumínica, ya que el tema residuos sigue siendo principalmente un tema manejado de modo sectorial por la autoridad sanitaria, y fuera del contexto de la Ley 19.300). 
En los dieciséis años de la Ley 19.300, se ha elaborado y dictado un importante número de normas ambientales y planes de prevención y descontaminación. También la literatura sobre costos y beneficios de regulaciones y medidas ambientales ha comenzado a ser más común. Sin embargo, el tema de la conservación de recursos naturales renovables (flora, fauna y vegetación) no se maneja en el mismo contexto conceptual, en cuanto a la evaluación costo-beneficio, que los temas de contaminación.

En este trabajo se pretende visualizar dónde podría utilizarse potencialmente el análisis costo-beneficio a lo largo de todo el proceso de elaboración de normas y planes para convertirse en una herramienta realmente efectiva en la toma de decisiones. Este análisis se amplía incluso al caso del sistema de evaluación de impacto ambiental. Asimismo, se revisa cuál ha sido la experiencia práctica de los estudios de impacto económico y social de normativa ambiental que se han realizado a la fecha, para evaluar su utilidad y determinar lo que aún queda por mejorar.

\section{Contexto normativo}

Hasta la promulgación de la Ley 19.300, el proceso formal de dictación de normas ambientales, o de generación de planes de descontaminación, no consideraba la variable económica. En efecto, dicho proceso ni siquiera obedecía a un desarrollo sistemático y consistente; se realizaba sectorialmente, de forma independiente entre los distintos servicios públicos, y en algunos casos incluso por mera resolución ministerial y sin publicidad alguna.

Previo a que se dictase la ley, no existía un organismo coordinador ni tampoco un procedimiento definido para la dictación de normas. De esta forma, éstas se elaboraban mediante procesos sin transparencia, que no estaban abiertos a la participación ciudadana. Un ejemplo de lo anterior lo constituye la Resolución 1.215 del Ministerio de Salud, dictada el año 1978, la cual estableció las normas primarias de calidad ambiental para contaminantes atmosféricos. Esta norma fue dictada por dicho ministerio sin realizar consultas ni a la ciudadanía ni a los titulares de las fuentes que se verían afectadas. Más aún, la resolución fue una copia directa de la normativa de Estados Unidos, la que curiosamente era producto de un proceso técnico-político y transparente. 
La promulgación de la Ley 19.300 y los reglamentos asociados (D.S. N 93/1995 Reglamento para la Dictación de Normas de Calidad Ambiental y de Emisión, D.S. N 94/1995 Reglamento que Fija el Procedimiento y Etapas para Establecer Planes de Prevención y de Descontaminación) significó un enorme avance en este sentido, puesto que en ellos se estableció que la dictación de normas y planes de prevención o descontaminación (los que constituyen el "brazo armado" de las normas de calidad ambiental, ya que son el instrumento operativo para lograr el cumplimiento de las mismas en zonas latentes o saturadas) se debe realizar de forma intersectorial y pública, es decir, en un proceso coordinado por la Conama en el cual participan todos los organismos competentes del Estado y que está abierto a consulta ciudadana.

Conforme con esta ley, el procedimiento de elaboración de normas debe contemplar una serie de etapas claramente definidas. Entre ellas, se considera un análisis técnico y económico de la norma, el desarrollo de estudios científicos, las consultas a organismos competentes, tanto públicos como privados, el análisis de las observaciones formuladas en la etapa anterior y una adecuada publicidad. Adicionalmente, se establece que las normas deben ser dictadas por Decreto Supremo, es decir, directamente por el (o la) Presidente(a) de la República, no pudiendo hacerlo un ministerio de forma independiente.

En cuanto al análisis técnico y económico a realizarse durante la elaboración de las normas, el reglamento establece que "dicho estudio deberá evaluar los costos y beneficios para la población, ecosistemas o especies directamente afectadas o protegidas [en el caso de normas secundarias]; los costos y beneficios a el o los emisores que deberán cumplir la norma; y los costos y beneficios para el Estado como responsable de la fiscalización del cumplimiento de la norma”. Como puede verse, la incorporación del criterio económico a lo largo del proceso para la definición de las normas y planes constituye una indicación de que éste pasa a jugar un rol en la gestión ambiental.

\section{Espacios para el análisis costo-beneficio}

\subsection{Programa priorizado de normas}

En cuanto a la definición de cuáles son las normas que deben elaborarse, la Ley 19.300 establece que se debe seguir un programa priorizado de normas, en el cual el director ejecutivo de Conama 
(instancia técnica), luego de consultar a los órganos competentes del Estado y a las organizaciones privadas involucradas, propone un conjunto de normas cuyos proceso de dictación o revisión debería comenzar. Posteriormente, el Consejo de Ministros (instancia política) decide cuáles de estas normas deben ser elaboradas. Es decir, la selección de normas a desarrollar obedece en última instancia a un criterio político.

En esta etapa del proceso de decisión, un análisis costo-beneficio que compare las distintas normas propuestas podría usarse para definir dónde concentrar los (escasos) recursos existentes para su desarrollo. Considerando el lento desarrollo que han tenido las normas², parece razonable que en primer lugar nos concentremos en dictar aquellas que generan el mayor beneficio social neto, para luego continuar con las menos rentables socialmente. De esta forma, al momento de priorizar las normas, quienes toman las decisiones dispondrían de una herramienta para ordenar las distintas iniciativas.

Un caso concreto donde lo anterior ya se ha realizado lo constituye el estudio "Análisis de Antecedentes para Evaluación de Escenarios en la Elaboración de la Norma de Calidad Primaria de MP2,5” realizado para Conama por Dictuc y Gestión Ambiental Consultores con el objeto de definir, en base a un análisis costo-beneficio, si es conveniente introducir una norma de calidad ambiental para el material particulado fino $(\mathrm{MP2}, 5)$ o si es preferible hacer más estricta la norma de material particulado respirable (MP10) existente, como de hecho está estipulado que ocurra el año 2012 si no se dicta para ese plazo una norma de MP2,5. Los resultados del análisis mostraron que una norma de MP2,5 generará beneficios sociales netos con relación a la norma de MP10, lo que ha dado pie a Conama para avanzar hacia su elaboración y posterior dictación.

Es importante señalar que la comparación de distintas normas para su priorización mediante el análisis costo-beneficio no está exenta de complejidad práctica. Esto se debe a la frecuente escasez de la información que se requiere para elaborar dichos estudios, lo que aumenta la incertidumbre respecto de los resultados. Sin embargo, en el

\footnotetext{
${ }^{2}$ Desde 1996 hasta 2009 se han seleccionado 76 normas para ser elaboradas o revisadas, habiéndose promulgado sólo 29, es decir un promedio de dos normas promulgadas por año. Actualmente hay 36 normas "en proceso" y que llevan un promedio de cuatro años en elaboración.
} 
entendido de que este análisis no pretende constituirse en la única variable de decisión, sí mostrará una tendencia que contribuirá a adoptar una definición más informada.

\subsection{Normas de calidad ambiental}

La Ley 19.300 define, en su artículo 2, Norma Primaria de Calidad Ambiental como:

Aquella que establece los valores de las concentraciones y períodos, máximos o mínimos permisibles de elementos, compuestos, sustancias, derivados químicos o biológicos, energías, radiaciones, vibraciones, ruidos o combinación de ellos, cuya presencia o carencia en el ambiente pueda constituir un riesgo para la vida o la salud de la población.

Como se puede ver, el concepto detrás de esta norma es el de minimizar los riesgos para la población. No obstante, para muchos contaminantes, como el material particulado, no existen niveles de concentración (umbrales mínimos) que permitan asegurar un nivel de riesgo nulo. Al mismo tiempo, en la ley no se definieron políticamente los niveles de riesgo que se aplicarían en Chile (por ejemplo, un riesgo de muerte de uno en uno millón o aquel equivalente a alguna actividad común). Un indicador de ese tipo habría hecho innecesaria la evaluación costo-beneficio, puesto que al contar con un nivel de riesgo definido se podría determinar el nivel de la norma simplemente recurriendo a la función de daño en salud (función que relaciona niveles de concentración de contaminantes con el incremento en el riesgo para la salud de las personas). En términos económicos, sólo restaría realizar un análisis de costo-efectividad para determinar cuáles son las medidas que permitirían alcanzar dicho nivel con el menor costo.

Sin embargo, al no existir niveles de riesgo definidos, el análisis costo-beneficio es el instrumento técnico que puede entregar información en base a la cual determinar los niveles apropiados para las normas de calidad ambiental. En particular, el nivel óptimo teórico de calidad ambiental debe ser aquel donde el beneficio marginal se iguala al costo marginal, puesto que para calidades ambientales mayores el costo de alcanzarlo comienza a ser mayor que el beneficio que éste genera (ver Figura $\mathrm{N}^{\circ} 1$ ). Si bien en la práctica los niveles teóricos óptimos son siempre muy difíciles, si es que no imposibles, de determinar, este 
FIGURA N ${ }^{\circ} 1: \quad$ NIVEL ÓPTIMO DE CALIDAD AMBIENTAL

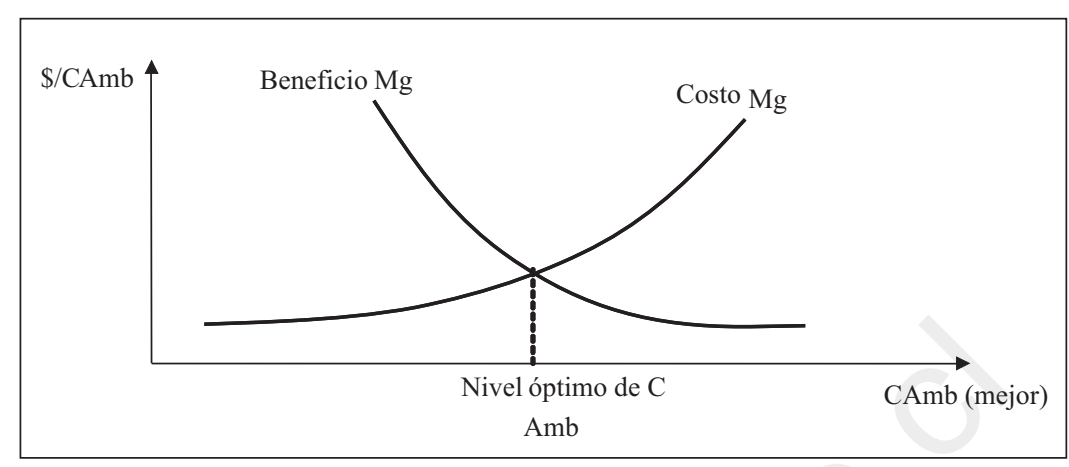

Fuente: Elaboración propia a partir de Kolstad, 1999.

análisis entrega el beneficio o costo social neto para el conjunto de alternativas evaluadas.

Por otra parte, la ley estableció que las normas primarias de calidad ambiental son de aplicación general en todo el territorio de la República, conforme al criterio de igualdad ante la ley, en este caso frente a los riesgos en salud asociados a la contaminación. Esta particularidad, de toda lógica en el plano legal y ético, genera complicaciones en el ámbito de una evaluación económica. Esto se debe a que, a igualdad de costos de control, los niveles "apropiados" de calidad ambiental que entrega un análisis de costo-beneficio son más estrictos cuando hay más personas afectadas, debido a que los beneficios totales (que corresponden a la suma de los beneficios individuales, y por ende es función del número de personas) serán mayores. Es más, los resultados son función de cómo se asignan los costos y beneficios a distintos grupos de personas. Al tener que definir un nivel de la norma igual para todo el país, la metodología se enfrenta a la dificultad de tener que poner en una misma balanza pequeños poblados y grandes ciudades.

\subsection{Normas de emisión}

Las normas de emisión establecen límites a la cantidad de contaminantes emitidos al aire o al agua que pueden producir las fuentes emisoras en general. El objetivo de estas normas puede ser la preven- 
ción de la contaminación o de sus efectos, o bien ser un medio para restablecer los niveles de calidad del aire o del agua cuando éstos han sido sobrepasados (en el contexto de planes de prevención o descontaminación). Su aplicación puede ser a nivel nacional o a nivel local, dependiendo del objetivo de protección que tenga la norma.

Conceptualmente, es razonable pensar que cada norma de emisión debería estar asociada a una norma de calidad, puesto que los efectos sobre las personas o el medio ambiente están directamente relacionados con las concentraciones ambientales de los distintos contaminantes (lo que define la segunda norma) y sólo indirectamente con la emisión desde las fuentes (lo que controla la primera norma). En efecto, éste ha sido el caso de varias de las normas dictadas de este tipo, como las normas de emisión de vehículos motorizados (D.S. 131/

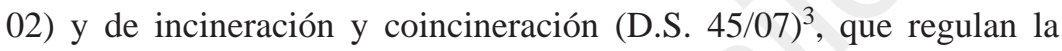
emisión de contaminantes atmosféricos para los cuales existen normas de calidad ambiental (MP, NOx, CO) a nivel de todo el país, es decir, no en el contexto de un plan de prevención o descontaminación.

Al existir un objetivo de calidad ya definido en la norma de calidad, el ejercicio económico que debiera realizarse para estas normas de emisión es el de comparar distintas alternativas para determinar con cuál de ellas se puede lograr una reducción de la concentración ambiental al mínimo costo, es decir, un análisis de costo-efectividad entre las distintas opciones de que se dispone. Este análisis permitiría desarrollar e implementar primero aquellas normas con el menor costo de reducción por unidad de emisión.

Por otra parte, cabe señalar que en la práctica también se han dictado normas de emisión que no tienen normas de calidad ambiental asociadas. Éste ha sido el caso de las normas de contaminación lumínica (D.S. 686/98), de olores molestos (D.S. 167/99), de riles a aguas superficiales (D.S. 90/00), de riles a aguas subterráneas (D.S. 46/02) y de ruido desde fuentes fijas (D.S. 146/97). En este caso, al no contar con normas que definan los niveles objetivo de calidad ambiental, se debe ir más allá de los costos. De esta forma, sí tiene sentido la realización de un análisis costo-beneficio, el que nos permitiría determinar desde qué niveles de la norma los costos sociales empiezan a superar a los beneficios.

${ }^{3}$ De especial relevancia es el proceso actualmente en desarrollo de la norma de emisión para centrales termoeléctricas. 


\subsection{Planes de descontaminación y prevención}

De forma análoga al caso de las normas de emisión que están asociadas a una norma de calidad, dado que los planes de descontaminación y prevención son instrumentos que buscan alcanzar ciertas metas de concentración ambiental, para cada uno de ellos bastaría con el desarrollo de un análisis costo-efectividad que comparara las distintas medidas identificadas para reducir las emisiones. De esta forma, deberían implementarse aquellas de menor a mayor costo, hasta que la reducción de emisiones acumulada permita devolver la zona saturada o latente a niveles bajo la norma ${ }^{4}$.

En efecto, el reglamento que fija el procedimiento de los planes de descontaminación establece que debe realizarse una "estimación del impacto social y económico" de la implementación de las distintas medidas. Es decir, se refiere a una cuantificación de los costos y no necesariamente de los beneficios, puesto que, al tener definida la concentración objetivo que se quiere alcanzar (según lo fije la norma de calidad ambiental), la evaluación de beneficios es redundante.

Por otra parte, el análisis costo-beneficio se podría utilizar para priorizar entre los distintos planes de prevención y descontaminación a desarrollar e implementar (en el sentido de analizar cuáles de ellos presentarían los mayores beneficios sociales netos), puesto que también en este caso la escasez de recursos no permite elaborarlos todos al mismo tiempo.

\subsection{El caso del SEIA}

La Ley 19.300 también creó el Sistema de Evaluación de Impacto Ambiental (SEIA), donde se establece el procedimiento de evalua-

\footnotetext{
${ }^{4}$ Respecto de este punto, cabe hacer mención que la Ley 19.300 establece en su artículo 45, letra $\mathrm{f}$, que en un plan de descontaminación la proporción en que las distintas fuentes deberán reducir sus emisiones deberá ser igual para todas ellas. Es decir, por más que la reducción de emisiones desde una fuente tenga un costo-efectividad mucho mayor que otra, ambas deberán reducir sus emisiones en el mismo porcentaje, lo que haría del análisis costo-efectividad un ejercicio no aplicable en la práctica. Sin embargo, este problema puede ser solucionado mediante la puesta en marcha de un sistema de transacción de cupos de emisión, mediante el cual todas las fuentes pueden acreditar reducciones proporcionales de emisiones, pero no necesariamente en su fuente. En ese escenario el sistema de precios priorizará automáticamente las medidas a implementar, comenzando por aquellas que tienen el menor costo marginal.
} 
ción ambiental de proyectos. Conforme la ley lo dispone, los proyectos serán aprobados solamente si cumplen con la normativa ambiental vigente y si se hacen cargo de sus impactos. Para lograr esto último, los proyectos deben proponer medidas de mitigación, compensación o reparación adecuadas, las que se definen como:

- $\quad$ Mitigación: medidas que tienen por finalidad evitar o disminuir los efectos adversos identificados.

- $\quad$ Reparación: medidas que tienen por finalidad reponer uno o más de los componentes o elementos del medio ambiente a una calidad similar a la que tenían con anterioridad al daño causado o, en caso de no ser ello posible, restablecer sus propiedades básicas.

- $\quad$ Compensación: medidas que tienen por finalidad producir o generar un efecto positivo alternativo y equivalente a un efecto adverso identificado, mediante el reemplazo o sustitución de los recursos naturales o elementos del medio ambiente afectados, por otros de similares características, clase, naturaleza y calidad.

Como se puede ver, estas medidas son en "especies ambientales” y no económicas. Es decir, el SEIA no permite el uso de transacciones monetarias (pagos) para hacerse cargo de los impactos. Esta manera de mitigar/reparar/compensar no permite una comparación directa con los beneficios económicos del proyecto, ya que estos últimos no se contrastan con los costos ambientales (salvo las mejoras en mejoramiento de empleos). Por ello, con relación a los efectos de los proyectos, existen dos canales paralelos, puesto que mientras los costos ambientales son sociales, los beneficios económicos son privados. No debe sorprender entonces que la aprobación de proyectos genere tanta oposición de parte de las comunidades locales que tendrán que soportar los efectos negativos de éstos sin recibir beneficios tangibles a cambio.

En este sentido, es interesante el proyecto de ley que estaría preparando el gobierno para generar un mecanismo de compensación económica para las comunas donde se instalen proyectos de centrales generadoras de energía. Estas compensaciones deberían ser establecidas siguiendo algún tipo de lineamiento con los impactos ambientales de las centrales (costos) de manera que no se generen distorsiones ambientales. 
Por otra parte, durante el proceso de generación de la Ley 19.300, la discusión sobre qué constituía contaminación fue el tema más largamente tratado. Finalmente, la ley definió contaminación como "la presencia en el ambiente de sustancias, elementos, energía o combinación de ellos, en concentraciones y permanencia superiores o inferiores, según corresponda, a las establecidas en la legislación vigente”. Es decir, la contaminación está definida en Chile como excedencia de una norma. Pero, ¿qué pasa cuando no hay normas?

En el contexto del SEIA, los artículos 6 y 7 de su reglamento indican que deben usarse normas de referencia, esto es, normas de otros países ${ }^{5}$. Este elemento genera una asimetría con relación a las normas dictadas en Chile puesto que, mientras para la aplicación de estas últimas se debe pasar por el procedimiento anteriormente descrito, la "importación” de las normas extranjeras para proyectos específicos se realiza sin ninguna evaluación económica o técnica. Además, la mayor parte de los países que se mencionan en el artículo 7 corresponde a países desarrollados, cuyos niveles de ingreso per cápita son mayores que el chileno. Como consecuencia, los beneficios que se habrían considerado en la definición de dichos límites son también muy superiores a los que se obtendrían en Chile, por lo que es de esperar que en estos países estén dispuestos a adoptar límites de concentraciones ambientales más estrictos (y por ende más costosos) que los que estaríamos dispuestos a adoptar en nuestro país si desarrolláramos una normativa local para dicho contaminante.

\section{El análisis costo-beneficio en la práctica: los AGIES}

La legislación exige la realización de un Análisis General del Impacto Económico y Social (AGIES) como parte del procedimiento de dictación de las normas de calidad ambiental, normas de emisión y planes de prevención y descontaminación. En esta sección se analiza cómo ha operado este instrumento como parte de la elaboración de las distintas regulaciones ambientales.

Los respectivos reglamentos (de normas y planes) establecen que el AGIES se debe elaborar luego de la publicación del anteproyecto.

${ }^{5}$ El artículo 7 indica que se podrán considerar los siguientes países: Alemania, Argentina, Australia, Brasil, Canadá, España, México, Estados Unidos, Nueva Zelanda, Países Bajos, Italia, Japón, Suecia y Suiza. 
Afortunadamente, en la práctica la tendencia ha sido elaborarlos en paralelo. Esto tiene la ventaja de permitir introducir la variable económica al momento de establecer los valores de las normas (o medidas, en el caso de los planes), antes de que éstos sean sometidos a consulta pública, lo que ocurre recién, y por única vez, cuando el anteproyecto correspondiente es publicado. Es decir, de esta forma el criterio económico pasa a ser un elemento de decisión en las fases iniciales de la elaboración de las regulaciones y no un simple elemento informativo hacia el final del proceso.

Conama generó metodologías para la elaboración de los AGIES (Conama, 1998) qué entregan un marco general, pero no permiten estandarizar metodologías de cálculo ni valores de costo-beneficio, lo que se refleja en los AGIES realizados a la fecha ${ }^{6}$, donde los costos y beneficios identificados y evaluados son muy diversos (ver Tabla $\mathrm{N}^{\circ} 1$ ). Incluso ciertos costos y beneficios definidos como no cuantificables en algunos AGIES han sido cuantificados en otros. Un ejemplo de ello corresponde al "valor de no uso", el cual no fue cuantificado en el AGIES de la norma de calidad secundaria del río Maipo pero sí lo fue en el del río Loa.

De la revisión de los AGIES desarrollados a la fecha se desprende además que los cálculos de costos y beneficios se realizan caso a caso, no existiendo valores estandarizados como aquellos con los que se cuenta para la evaluación social de proyectos en el Ministerio de Planificación. El único valor estandarizado, y que se ha utilizado de forma más consistente, corresponde a la tasa de descuento social, utilizada para el cálculo de los valores presentes.

Adicionalmente, en varios de los AGIES analizados no se concluye respecto del beneficio social neto, es decir, no se llega a un valor último de la evaluación. Sólo se cuantifican algunos costos y beneficios

${ }^{6}$ Como parte de este trabajo se revisaron los AGIES de las siguientes normas (se indica el decreto supremo correspondiente para aquellas que ya han sido dictadas): Norma de Calidad Primaria para Material Particulado Respirable (D.S. 45/01), para Plomo (D.S. 136/00) y para Dióxido de Azufre (D.S. 113/02), Norma de Calidad Secundaria del río Loa y del río Maipo, Revisión de la Norma de Emisión de Ruidos Molestos (D.S. 46/97), Norma de Emisión de Arsénico al Aire (D.S. 165/99), Norma de Emisión para la Regulación de Contaminación Lumínica (D.S. 686/98), Norma de Emisión de Vehículos Motorizados (D.S. 131/02), de Motocicletas (D.S. 103/00) y de Grupos Electrógenos y las Normas de Emisión de Riles a Aguas Superficiales (D.S. 90/00), a Aguas Subterráneas (D.S. 46/02) y a Alcantarillado (D.S. 609/98). 
TABLA N ${ }^{\circ}$ 1: $\quad$ COSTOS Y BENEFICIOS CONSIDERADOS EN LOS AGIES DE DISTINTAS NORMAS

\begin{tabular}{|c|c|c|c|c|c|}
\hline & & $\begin{array}{l}\text { NPCA } \\
\text { MP10 }\end{array}$ & $\begin{array}{l}\text { NPCA } \\
\text { Plomo }\end{array}$ & $\begin{array}{l}\text { NPCA } \\
\mathrm{SO}_{2}\end{array}$ & $\begin{array}{c}\mathrm{NE} \\
\text { Generadores }\end{array}$ \\
\hline Costos & $\begin{array}{l}\text { Abatimiento } \\
\text { Monitoreo } \\
\text { Fiscalización } \\
\text { Disminución capa- } \\
\text { cidad sist. transp. } \\
\text { Vida útil equipos }\end{array}$ & $\mathrm{X}$ & $\begin{array}{l}X \\
X\end{array}$ & $\mathrm{X}$ & $\begin{array}{l}X \\
X \\
X\end{array}$ \\
\hline Beneficios & $\begin{array}{l}\text { Salud } \\
\text { Tiempo de viaje } \\
\text { Reforzamiento escolar } \\
\text { Materiales } \\
\text { Visibilidad }\end{array}$ & $\begin{array}{l}X \\
X\end{array}$ & $\begin{array}{l}X \\
X\end{array}$ & $\mathrm{X}$ & $\mathrm{X}$ \\
\hline
\end{tabular}

NPCA: Norma Primaria de Calidad Ambiental.

NE: Norma de Emisión.

Fuente: Elaboración propia.

de forma aislada, pero sin llegar a un resultado que ayude a la toma de decisiones. Un ejemplo de ello corresponde al AGIES de la norma de emisión de riles a aguas superficiales (DS N 90/00).

En el caso de algunos AGIES, incluso se ha evidenciado que ningún costo o beneficio es cuantificado, y el análisis se limita al ámbito cualitativo. Tal es el caso de los AGIES de la norma de emisión para vehículos motorizados (D.S. 131/02), de la norma de emisión de riles a aguas subterráneas (D.S. 46/02) y de la norma de calidad secundaria del río Loa (en elaboración).

Varias de las carencias mencionadas anteriormente probablemente tienen su origen en la baja cantidad de recursos que se han destinado a este tipo de estudios. Éstos han contado con un presupuesto medio de sólo US\$ 15.000, según información entregada por Conama.

Cabe señalar además que, a pesar de que la ley establece que los procesos de elaboración de normas deben contar con expedientes públicos, dentro de los cuales se debería encontrar el respectivo AGIES, esto no siempre ocurre en la actualidad. Durante el desarrollo del presente trabajo fueron pocos los AGIES que se encontraron disponi- 
bles, puesto que muchos expedientes están extraviados o inubicables en las oficinas de Conama.

Finalmente, y más allá de las debilidades descritas sobre los estudios económicos de las distintas normas y planes, es importante valorar su incorporación en estos procesos. En este sentido, cabe notar que en la gestión de otros componentes ambientales estos estudios están completamente ausentes. Tal es el caso de la gestión de recursos naturales, donde sólo se utilizan criterios ecológicos (ejemplos de ello son las definiciones de áreas silvestres protegidas y de los listados de especies en categorías de conservación). Cabe preguntarse entonces si estos componentes del medio ambiente tienen mayor valor intrínseco que la protección de la salud de la población y si es por ello que no interesa la variable costo.

\section{Conclusiones y recomendaciones}

La Ley 19.300 significó un avance importante en la generación de normas ambientales en Chile. La nueva institucionalidad cambió la forma de generar regulaciones, estableciendo procedimientos claros, transparencia y participación ciudadana. Asimismo, introdujo el criterio económico en la gestión ambiental.

Es interesante que en dicha ley no se haya definido políticamente el nivel de riesgo aceptable, quedando éste como un aspecto en el cual no hay responsabilidades.

Por otra parte, existen temas éticos relacionados con los niveles de ingreso. En el caso de varias normas dictadas en Chile, se han importado directamente las normas de países desarrollados en los cuales los costos podrían ser iguales, pero no los beneficios (conforme metodologías económicas comúnmente aceptadas), lo que está relacionado con el nivel de ingreso per cápita.

Del análisis de los AGIES se concluyó que la metodología utilizada en las evaluaciones no ha sido homogénea, y en ellas hay muy diversos costos y beneficios considerados y cuantificados. Asimismo, en el caso de varios de ellos se genera una importante subjetividad por la existencia de variables "no cuantificadas".

En definitiva, y a la luz de lo que se ha realizado en la práctica, el criterio económico sigue siendo un elemento relativamente "externo" en 
la toma de decisiones ambientales. Por ello, a modo de recomendaciones para maximizar los beneficios que puede entregar esta herramienta, se propone lo siguiente:

- El análisis costo-beneficio se puede utilizar como instrumento para priorizar el cronograma de implementación de normas y planes.

- $\quad$ En el caso de las normas de emisión con una meta ambiental asociada (i. e. una norma de calidad ambiental), debería realizarse un análisis de costo-efectividad entre las alternativas de control. En el caso de las normas sin meta de calidad asociada, se deberían evaluar mediante análisis costo-beneficio.

- $\quad$ Debiera formalizarse el hecho de que el AGIES debe realizarse antes y no después del anteproyecto de las normas.

- $\quad$ Se debe estandarizar la metodología para elaborar los AGIES, teniendo claramente definidas las variables que se considerarán y homogeneizados los costos para los elementos más relevantes.

- $\quad$ Se debe incorporar la variable económica en la gestión de otros componentes ambientales, en particular en la gestión de recursos naturales.

Finalmente, es importante tener presente que el análisis costobeneficio debe ser entendido como un insumo para la decisión y nunca como una condición vinculante. En la definición de los estándares de calidad ambiental que como sociedad buscamos imponernos, ni éste ni otros criterios técnicos deben sobreponerse a la decisión política.

\section{REFERENCIAS}

Calfucura, Enrique (2006): “Análisis técnico-económico del anteproyecto de norma de emisión de material particulado y gases para grupos electrógenos en la Región Metropolitana”. Elaborado para Conama Región Metropolitana.

Conama (1998): "Metodologías para el Estudio de los Efectos Económicos y Sociales de Planes y Normas Ambientales".

(2007): “Análisis del impacto económico y social del anteproyecto de revisión del D.S. N 146/97 MINSEGPRES norma de emisión de ruidos molestos generados por fuentes fijas".

Conama, Departamento de Instrumentos y Análisis Económico (2001): “Análisis general de impacto económico y social del anteproyecto de norma de emisión de residuos líquidos a aguas subterráneas”. 
(2001): “Análisis general del impacto económico y social del anteproyecto de revisión de la norma de emisión de contaminantes aplicables a los vehículos motorizados establecida por el D.S. No 4 de 1994 del Ministerio de Transporte y Telecomunicaciones".

Conama, Región Metropolitana (2000): “Análisis general del impacto económico y social anteproyecto de norma nacional de emisión para motocicletas”.

Conama, Unidad de Economía Ambiental (1997): “Análisis general del impacto económico y social anteproyecto de norma para la regulación de contaminantes asociados a las descargas de residuos líquidos a aguas superficiales”.

- (1997): “Análisis general del impacto económico y social norma de emisión para la regulación de contaminantes asociados a las descargas de residuos industriales líquidos a sistemas de alcantarillados”.

- (1998): “Análisis general del impacto económico y social anteproyecto de norma de emisión para la regulación del contaminante arsénico emitido al aire".

- (1998): “Análisis general del impacto económico y social del anteproyecto de norma de emisión para la regulación de la contaminación lumínica”.

(1999): “Análisis general del impacto económico y social del anteproyecto de revisión de la norma de calidad primaria para material particulado respirable, MP10”.

(2000): “Análisis general del impacto económico y social anteproyecto de norma de calidad primaria para plomo en el aire”.

Conama (s/f): "Análisis general del impacto económico y social de las normas de calidad primaria de aire de partículas totales en suspensión (PTS), Ozono (O3), anhídrido sulfuroso (SO2), monóxido de carbono (CO) y dióxido de nitrógeno (NO2)".

Dictuc-Gestión Ambiental Consultores (2007): “Análisis de antecedentes para evaluación de escenarios en la elaboración de la norma de calidad primaria de MP2,5”. Elaborado para Conama.

Econat Consultores (2005): “Análisis general del impacto económico de norma secundaria de calidad de aguas del río Loa en el sector silvoagropecuario”. Elaborado para SAG Región de Antofagasta.

Kolstad, Charles (1999): Environmental Economics. Oxford University Press. 